\title{
Large scale evaluation of $\beta$-decay rates of r-process nuclei
}

\section{T. Marketin*}

Department of Physics, Faculty of Science, University of Zagreb, 10000 Zagreb, Croatia

E-mail: market inephy.hr

\section{Huther}

Institut für Kernphysik (Theoriezentrum), Technische Universität Darmstadt, 64289 Darmstadt, Germany

\section{G. Martínez-Pinedo}

Institut für Kernphysik (Theoriezentrum), Technische Universität Darmstadt, 64289 Darmstadt, Germany

GSI Helmholtzzentrum für Schwerionenforschung, Planckstraße 1, 64291 Darmstadt, Germany

\begin{abstract}
R-process nucleosynthesis models rely, by necessity, on nuclear structure models for input. Particularly important are the $\beta$-decay half-lives of neutron-rich nuclei which have a direct impact on the distribution of elemental abundances. The interacting shell model has been applied to the calculation of decay properties of nuclei, but due to the computational cost, it is restricted to the nuclei around the closed shells leaving the quasiparticle random phase approximation (QRPA) as the only approach able to provide results across the whole nuclear chart. However, currently only a single large-scale calculation of $\beta$-decay properties exists, and it is based on a QRPA calculation with a schematic interaction on top of the Finite Range Droplet Model. In this work we utilize a fully self-consistent theoretical framework based on the relativistic Hartree-Bogoliubov (RHB) model + QRPA in order to study both the allowed and the first-forbidden transitions in several thousand nuclei involved in the r-process nucleosynthesis. The transition rates, together with the obtained $\beta$-delayed neutron emission probabilities are then employed in the r-process simulations with the goal of obtaining the resulting elemental abundaances.
\end{abstract}

XIII Nuclei in the Cosmos,

7-11 July, 2014

Debrecen, Hungary

\footnotetext{
* Speaker.
} 


\section{Introduction}

The creation of heavy elements is a complex and delicate process spanning the whole nuclear chart. While the actual site of the r-process has yet to be identified, it is clear that it involves thousands of nuclei from the valley of stability to the neutron drip-line, in an environment with relatively high temperatures (up to $1 \mathrm{GK}$ ) and neutron densities $\left(n_{n}>10^{20} \mathrm{~g} \mathrm{~cm}^{-3}\right)$. Due to the limitations of the current experimental facilities, and the impractical nature of measuring different physical properties of such a large number of nuclei, it falls on theory to provide the necessary input for the r-process nucleosynthesis simulations. The r-process poses a particularly challenging problem because it requires input for many nuclear processes. Of those, $\beta$-decay and the $\beta$-delayed neutron emission probabilities are very important due to their influence on the final distribution of abundances [四]. Additionally, because r-process simulations need input on many different properties of nuclei, it is necessary to employ microscopic, self-consistent models that can be just as reliably applied near the neutron drip-line, as they can be close to the valley of stability. In the present work, we describe the application of a fully self-consistent theoretical framework based on the relativistic nuclear energy density functional (RNEDF) [D].

\section{Theoretical formalism and results}

The calculation of $\beta$-decay rates requires the evaluation of both the nuclear ground state of the parent nucleus and the excited states of daughter nucleus, together with the transitions between them, along with the determination of the lepton phase space involved in the transition. Due to these requirements, and the application of the formalism to a very large nuber of nuclei, we employ a fully microscopic theoretical framework [3] based on the relativistic nuclear energy density functional (RNEDF). The ground state is constructed with the relativistic Hartree-Bogoliubov model which properly describes the pairing in open shell nuclei. Assuming spherical symmetry of the nuclear system, the excited states are obtained by solving the QRPA equations

$$
\left(\begin{array}{cc}
A & B \\
B^{*} & A^{*}
\end{array}\right)\left(\begin{array}{l}
X^{\lambda} \\
Y^{\lambda}
\end{array}\right)=E_{\lambda}\left(\begin{array}{cc}
1 & 0 \\
0 & -1
\end{array}\right)\left(\begin{array}{l}
X^{\lambda} \\
Y^{\lambda}
\end{array}\right),
$$

where the residual interaction is derived from the same density functional as the one used at the ground state level [四].

The decay is determined by the phase space available to the leptons, the Fermi function which accounts for the changes of the outgoing electron wave function and the shape factor which contains the nuclear physics. The decay rate reads

$$
\lambda_{i}=D \int_{1}^{W_{0, i}} W \sqrt{W^{2}-1}\left(W_{0, i}-W\right)^{2} F(Z, W) C(W) d W
$$

where the constant $D$, and the half-life are

$$
T_{1 / 2}=\frac{\ln 2}{\lambda}, \quad D=\frac{\left(G_{F} V_{u d}\right)^{2}}{2 \pi^{3}} \frac{\left(m_{e} c^{2}\right)^{5}}{\hbar} .
$$

The critical component of the evaluation of the decay rates is the shape factor $C(W)$, which for allowed decays is equal to the Gamow-Teller transition strength, but for the first-forbidden transitions involves a complex combination af transition matrix elements and the electron energy [ [5, 6]. 


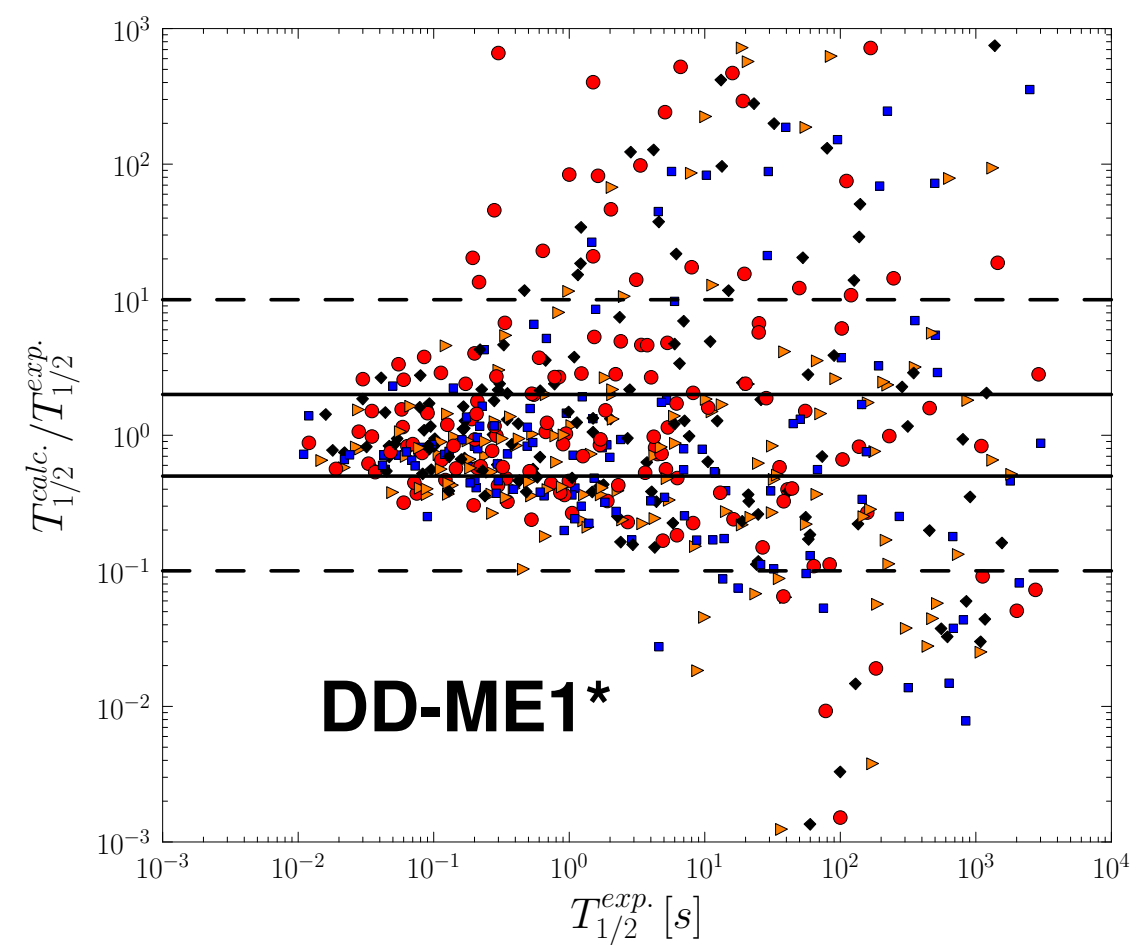

Figure 1: The ratio of the calculated and the experimental half-lives versus the experimental half-lives. The even-even nuclei are denoted with blue squares, odd-N nuclei with orange triangles, odd-Z nuclei with black diamonds and the odd-odd nuclei with the red circles. The data is taken from the AME 2012 data table. [D]

In Fig. Wwe plot the ratio of the calculated and experimental half-lives versus the experimental half-lives for nuclei with half-lives shorter than 1h included in the AME 2012 [ [ ] ] nuclear data evaluation. The description of long-lived nuclei is challenging due to the very small $Q_{\beta}$ window which contains only a few transitions, thus making the decay rate very sensitive to the details of the strength distribution at the low-energy part of the spectrum. However, with the increase of the Q-values (and the decrease of the half-lives) the description of the decay rates is significantly improved. For nuclei with $T_{1 / 2}<1 \mathrm{~s}$, the half-lives of practically all of the nuclei are predicted within an order of magnitude, with most of them found within a factor of two. The half-lives of even-even nuclei and the odd-N nuclei tend to be underestimated, and the half-lives of odd- $Z$ and odd-odd nuclei tend to be overestimated, but the average deviation of all types of nuclei is within cca. $25 \%$ of the experimental value.

In addition to the $\beta$-decay rates, in the late stages of the r-process, when neutron-rich matter is decaying towards stability, the decay products may deexcite by emitting one or more neutrons, depending on the details of the decay and the neutron separation energies. In Fig. $\square$ we plot the average number of emitted neutrons per decay for the nuclei involved in the r-process. Close to the valley of stability, due to the low Q-values and high neutron separation energies, the chances of emitting even a single neutron are very low, and the average number of neutrons is close to 0 . However, for very neutron rich nuclei, both the Q-values are larger and the neutron separation energies are lower enabling the emission of multiple neutrons. Moving towards the neutron dripline, the average number of emitted neutrons saturates at values between 3 and 5 neutrons per 


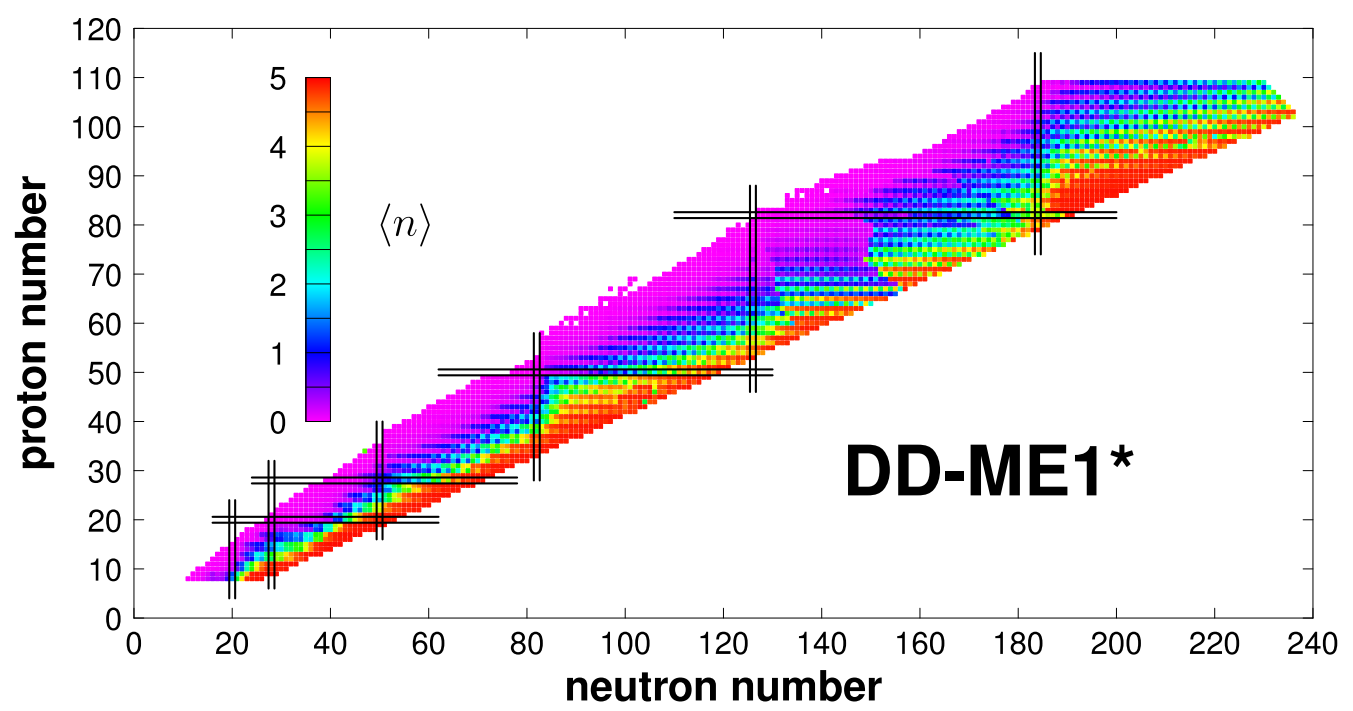

Figure 2: The average number of emitted neutrons after $\beta$-decay obtained from the present calculation.

decay, depending on the region of the nuclear chart.

Using the results of the present calculation in a cold r-process calculation, where the $(n, \gamma) \leftrightarrow$ $(\gamma, n)$ equillibrium is not reached, we observe noticeable differences in the resulting r-process abundances, when compared to the results obtained using the only existing data table of $\beta$-decay rates [ [8], as shown in Fig. [3]. Instead of focusing on a detailed analysis of the differing abundances, here we simply point out that weak-interaction rates are a very important ingredient of the r-process nucleosynthesis simulations. Combined with the uncertainties arising from different mass models, and other sources of nuclear input, the differences will increase even further and span a very wide range of possible end states of the r-process. This illustrates a pressing need for a self-consistent, fully microscopic model whch contains all the relevant physics and which could provide all the various requierd inputs within a singular theoretical framework.

\section{Conclusion}

In this study we have performed a large scale calculation of $\beta$-decay rates and $\beta$-delayed neutron emission probabilities, including both allowed and first-forbidden transitions treated on an equal footing, for neutron-rich nuclei with $8 \leq Z \leq 110$. The comparison of the results with the available data has shown that for the short-lived nuclei the model performs very well, with the majority of nuclei being found within a factor of two away from the experimental value. We have also evaluated the $\beta$-delayed neutron emission probabilities and by including them in an illustrative r-process nucleosynthesis simulation, we have observed a noticeable change in the resulting elemental abundances. This result points to a need for a unique, self-consistent, fully microscopic model that could provide all the relevant nuclear input required in astrophysical simulation, thus reducing one source of uncertainties. 


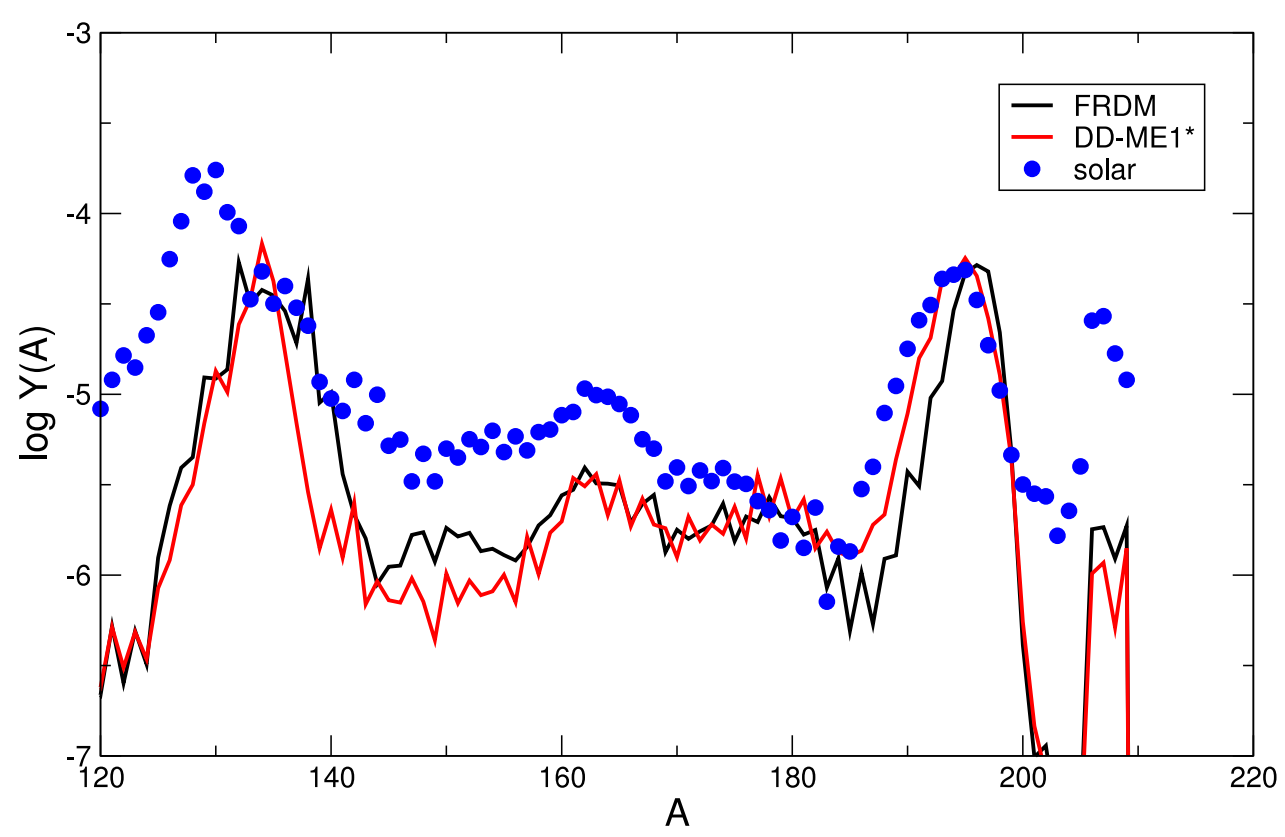

Figure 3: Comparison of elemental abundances obtained for a cold r-process calculation using the $\beta$-decay data from the FRDM data table and the present work.

\section{References}

[1] K. Langanke and G. Martínez-Pinedo, Reviews of Modern Physics 75, 819 (2003).

[2] D. Vretenar, A. Afanasjev, G. Lalazissis, and P. Ring, Physics Reports 409, 101 (2005).

[3] I. N. Borzov, Nuclear Physics A 777, 645 (2006).

[4] N. Paar, T. Nikšić, D. Vretenar, and P. Ring, Physical Review C 69, 054303 (2004).

[5] H. Behrens and W. Bühring, Nuclear Physics A 162, 111 (1971).

[6] Q. Zhi, E. Caurier, J. J. Cuenca-García, G. Martínez-Pinedo, and K. Sieja, Physical Review C 87, 025803 (2013).

[7] G. Audi, F. G. Kondev, M. Wang, B. Pfeiffer, X. Sun, J. Blachot, and M. MacCormick, Chinese Physics C 36, 1157 (2012).

[8] P. Möller, B. Pfeiffer, and K.-L. Kratz, Physical Review C 67, 055802 (2003). 